\title{
EUS-guided biliary drainage: A systematic review and meta-analysis
}

\author{
Banreet Singh Dhindsa ${ }^{1}$, Harmeet Singh Mashiana ${ }^{2}$, Amaninder Dhaliwal' ${ }^{2}$, Babu P. Mohan ${ }^{3}$, \\ Mahendran Jayaraj4, Harlan Sayles5, Shailender Singh ${ }^{2}$, Gordon Ohning ${ }^{4}$, Ishfaq Bhat ${ }^{2}$, Douglas G. Adler ${ }^{6}$ \\ ${ }^{1}$ Department of Internal Medicine, University of Nevada Las Vegas School of Medicine, ${ }^{4}$ Division of Gastroenterology, \\ University of Nevada Las Vegas School of Medicine, Las Vegas, Nevada, 'Division of Gastroenterology and Hepatology, \\ University of Nebraska Medical Center, ${ }^{5}$ Department of Biostatistics, University of Nebraska Medical Center, Omaha, \\ $\mathrm{NE}$, ${ }^{3}$ Banner University Medical Center, University of Arizona, Tucson, AZ, ${ }^{6} \mathrm{H} u n t s m a n$ Cancer Center, University of Utah \\ School of Medicine, Salt Lake City, Utah, USA
}

\begin{abstract}
ERCP is the current procedure of choice for patients with jaundice caused by biliary obstruction. EUS-guided biliary drainage (EUS-BD) has emerged as an alternative to ERCP in patients requiring biliary drainage. The aim of the study was to conduct a systematic review and meta-analysis to report the overall efficacy and safety of EUS-BD. We conducted a comprehensive search of several databases including PubMed, EMBASE, Web of Science, Google Scholar, and LILACS databases (earliest inception to June 2018) to identify studies that reported EUS-BD in patients. The primary outcome was to look at the technical and clinical success of the procedure. The secondary analysis focused on calculating the pooled rate of re-interventions and all adverse-events, along with the commonly reported adverse-event subtypes. Twenty-three studies reporting on 1437 patients were identified undergoing 1444 procedures. Majority of the patient population were male $(53.86 \%)$, with an average age of 67.22 years. The pooled technical success rates and clinical success rates were $91.5 \%$ (95\% confidence interval [CI]: 87.7-94.2, $\left.I^{2}=76.5\right)$ and $87 \%$ (95\% CI: 82.3-90.6, $\left.I^{2}=72.4\right)$, respectively. The total adverse event rates were $17.9 \%\left(95 \%\right.$ CI: $\left.14.3-22.2, I^{2}=69.1\right)$. Subgroup analysis of three major individual adverse events was bile leak: $4.1 \%\left(2.7-6.2, I^{2}=46.7\right)$, stent migration: $3.9 \%(2.5-6.2$, $\left.I^{2}=43.5\right)$, and infection: $3.8 \%\left(2.8-5.1, I^{2}=0\right)$ Substantial heterogeneity was noted in the analysis. EUS-BD has high technical and clinical success rate and hence a very effective procedure. Concerns about publication bias exist. Careful consideration should be given to the adverse events and weighing the risks and benefits of the alternative nonsurgical/ surgical approaches.
\end{abstract}

Key words: Biliary drainage, Biliary obstruction, ERCP, EUS guided, jaundice

\begin{tabular}{|l|l|}
\hline \multicolumn{2}{|c|}{ Access this article online } \\
\hline Quick Response Code: & Website: \\
\hline & www.eusjournal.com \\
\cline { 2 - 2 } & DoI: \\
\hline
\end{tabular}

This is an open access journal, and articles are distributed under the terms of the Creative Commons Attribution-NonCommercial-ShareAlike 4.0 License, which allows others to remix, tweak, and build upon the work non-commercially, as long as appropriate credit is given and the new creations are licensed under the identical terms.

For reprints contact: reprints@medknow.com

How to cite this article: Dhindsa BS, Mashiana HS, Dhaliwal A, Mohan BP, Jayaraj M, Sayles $\mathrm{H}$, et al. EUS-guided biliary drainage: A systematic review and meta-analysis. Endosc Ultrasound 2020;9:101-9.

\section{Address for correspondence}

Dr. Douglas G. Adler, Huntsman Cancer Center, School of Medicine, University of Utah, 30 N 1900 E, Room 4R118, Salt Lake City, Utah 84132, USA. E-mail: douglas.adler@hsc.utah.edu

Received: 2019-02-27; Accepted: 2019-12-25; Published online: 2020-04-15 


\section{INTRODUCTION}

ERCP is the current procedure of choice for patients with jaundice caused by biliary obstruction. It has a high success rate and a low major adverse event rate. ${ }^{[1]}$ Failure of ERCP can be secondary to surgically altered anatomy, inaccessible papilla due to malignancy, or (rarely) cannulation failure. Percutaneous drainage has historically been the treatment of choice in patients with failed ERCP.

EUS-biliary drainage (BD) was first described by Giovannini in 2001. ${ }^{[2]}$ Since then, EUS-BD has emerged as an alternative to ERCP in patients requiring $\mathrm{BD}$. Various studies have reported high technical success associated with EUS-BD.

The aim of this study was to evaluate the technical success, clinical success, and adverse events of EUS-BD.

\section{METHODS}

\section{Search strategy}

We conducted a comprehensive search of several databases and conference proceedings including PubMed, EMBASE, Web of Science, Google Scholar, and LILACS databases (earliest inception to June 2018). We followed the Preferred Reporting Items for Systematic Reviews and Meta-Analyses guidelines, using a predefined protocol, to identify studies reporting EUS-guided BD. An experienced medical librarian using inputs from the study authors helped with the literature search.

Keywords used in search included a combination of "Endoscopic ultrasound," "EUS," "biliary," “drainage," and "complications." The search was restricted to studies in human subjects and published in the English language in peer-reviewed journals. Two authors (BSD and HSM) independently reviewed the title and abstract of studies identified in primary search and excluded studies that did not address the research question, based on prespecified exclusion and inclusion criteria. The full text of the remaining articles was reviewed to determine whether it contained relevant information. Any discrepancy in article selection was resolved by consensus and in discussion with a coauthor.

The bibliographic section of the selected articles as well as the systematic and narrative articles on the topic was manually searched for additional relevant articles.
Study selection

In this meta-analysis, we included cohort studies that met the following criteria: (1) EUS-BD (2) information on adverse events, (3) information on subcategories of adverse events, (4) technical success rate, and (5) clinical success rate. Studies irrespective of the geography and abstract/manuscript status were included as long as they provided data needed for the analysis.

We excluded the studies that (1) provided insufficient data to allow the estimation of adverse events and (2) insufficient data on the subcategories of adverse events.

In case of multiple publications from the same cohort, data from the most recent comprehensive report were included. We did not encounter any such study in our analysis.

\section{Data abstraction and quality assessment}

Data on study-related outcomes in the individual studies were abstracted onto a standardized form by at least two authors (BSD and HSM) independently, and two authors (BSD and HSM) did the quality scoring independently.

Using a scale modified from the Newcastle-Ottawa scale for cohort studies assessed the quality of the included studies. ${ }^{[3]}$ This quality score consisted of three categories: (1) selection - included four questions and maximum one star per question could be awarded (2) comparability - included one question and maximum two stars could be awarded, and (3) exposure - included three questions and maximum one star per question could be awarded. Good-quality study was defined as 3 or 4 stars in the selection domain and 1 or 2 stars in comparability domain and 2 or 3 stars in the outcome domain. Fair-quality study was defined as 2 stars in the selection domain and 1 or 2 stars in comparability domain and 2 or 3 stars in the outcome domain. Poor-quality study was defined as 0 or 1 star in the selection domain and 0 stars in comparability domain and 0 or 1 star in the outcome domain.

Quality assessment for randomized controlled trials (RCTs) was done with Jadad-Oxford scale. A maximum of 5 points could be given to a study on the basis of randomization, blinding, and withdrawals from the study. A score of $\leq 2$ was defined as a poor-quality study. 


\section{Outcomes assessed}

The primary analysis of this study focused on calculating the pooled rate of overall technical success and clinical success in EUS-BD.

Our secondary analysis focused on calculating the pooled rate of re-interventions and all adverse events, along with the commonly reported adverse-event subtypes (pneumoperitoneum, infection, bleeding, bile leak, and stent migration).

\section{Statistical analysis}

We used meta-analysis techniques to calculate the pooled estimated in each case following the methods suggested by DerSimonian and Laird using the random-effect model, and our application can be seen to fit within their general approach (where the effect is measured by the probability of risk). ${ }^{[4]}$ When the incidence of an outcome was zero in a study, a correction of 0.5 was added to the number of incident cases before the statistical analysis. ${ }^{[5]}$ We assessed heterogeneity between study-specific estimates using two methods: Cochrane's Q statistics and $I^{2}$ statistics. ${ }^{[6,7]}$ In this, values of $<30 \%$, $30 \%-60 \%, 61 \%-75 \%$, and $>75 \%$ were suggestive of low, moderate, substantial, and considerable heterogeneity, respectively. ${ }^{[8]}$ Publication bias was ascertained qualitatively by visual inspection of funnel plot and quantitatively by the Egger test. ${ }^{[0]}$ When publication bias was present, further statistics using the fail-Safe $\mathrm{N}$ test and Duval and Tweedie's "Trim and Fill test" was used to ascertain the impact of the bias. ${ }^{[10]}$ Three levels of impact were reported based on the concordance between the reported results and the actual estimate if there was no bias. The impact was reported as minimal if both versions were estimated to be the same; modest if effect size changed substantially, but the final finding would still remain the same; and severe if the basic final conclusion of the analysis is threatened by the bias. ${ }^{[1]}$

All analyses were performed using Comprehensive Meta-Analysis software, version 3 (BioStat, Englewood, NJ, USA).

\section{RESULTS}

\section{Search results and population characteristics}

From a total of 761 citations identified by our search criteria, 47 studies reported the use of EUS-BD.

The schematic diagram of the study selection is illustrated in Supplementary Figure 1.
Table 1 describes the study characteristics. Majority of the patient population were males $(53.86 \%)$, with an average age of 67.22 years. The indications for the EUS-BD included malignant obstructive jaundice, cholangitis, bile leak, and other benign causes. In the majority of studies, technical success was defined as successful deployment of the stent and clinical success was defined as a reduction in serum total bilirubin by $50 \%$ at 1 week or $<3 \mathrm{mg} / \mathrm{dL}$ at 2 weeks after the procedure.

The type of intervention during the EUS was through transgastric (29.5\%), transduodenal (34.34\%), and other techniques $(19.8 \%)$. One of the studies did not mention the technique used, so the above numbers exclude that study.

\section{Characteristics and quality of the included studies}

Table 1 describes the characteristics of the included studies.

The meta-analysis included 20 independent cohort studies and 3 RCTs with a total of 1437 patients.

None of the studies were population based. Ten studies were multicenter based, and the rest were from single center. Thirteen studies had more than 40 patients, 3 studies had 30-40 patients, and 7 studies had $<30$ patients. All of the included studies had clear information reported on the technical success, clinical success, and adverse-event rates, including the subcategory of the adverse events. All the studies were published in the original manuscript form. Overall, 18 studies were considered to be of high quality and rest 5 were considered medium quality. None were of low quality.

Supplementary Table 1 details the study quality assessment.

\section{Technical success and clinical success}

The calculated pooled rate of technical success was 91.5\% (95\% confidence interval [CI]: 87.7-94.2, $\left.I^{2}=76.5\right)$.

The calculated pooled rate of clinical success was 87\% (95\% CI: 82.3-90.6, $\left.I^{2}=72.4\right)$.

Figures 1 and 2 show the forest plot for technical and clinical success, respectively. 
Table 1. Study description and clinical outcomes

\begin{tabular}{|c|c|c|c|c|c|c|c|c|c|c|}
\hline & \multicolumn{2}{|c|}{$\begin{array}{l}\text { Study } \\
\text { year }\end{array}$} & Study type & \multicolumn{2}{|c|}{$\begin{array}{l}\text { Single center } \\
\text { or multicenter }\end{array}$} & $\begin{array}{l}\text { Abstract or } \\
\text { manuscript }\end{array}$ & $\begin{array}{l}\text { Cohort/case } \\
\text { control/RCT }\end{array}$ & \multicolumn{2}{|c|}{$\begin{array}{c}\text { Number of } \\
\text { patients }\end{array}$} & $\begin{array}{c}\text { Mean } \\
\text { age }\end{array}$ \\
\hline Minaga et al..$^{[12]}$ & \multicolumn{2}{|c|}{2019} & Prospective & \multicolumn{2}{|c|}{ Multicenter } & Manuscript & RCT & \multicolumn{2}{|c|}{47} & 73 \\
\hline Park et al. ${ }^{[13]}$ & \multicolumn{2}{|c|}{2018} & Prospective & \multicolumn{2}{|l|}{ Single } & Manuscript & $\mathrm{RCT}$ & \multicolumn{2}{|c|}{14} & 66.8 \\
\hline Tsuchiya et al. ${ }^{[14]}$ & \multicolumn{2}{|c|}{2018} & Prospective & \multicolumn{2}{|c|}{ Multicenter } & Manuscript & Cohort & \multicolumn{2}{|c|}{19} & 70.6 \\
\hline Kahaleh et al..$^{[15]}$ & & 16 & Retrospective & Multicen & ter & Manuscript & Cohort & 3 & & 81 \\
\hline Tyberg et al..$^{[16]}$ & & 16 & Prospective & Single & & Manuscript & Cohort & 5 & & 68 \\
\hline Khashab et al..$^{[17]}$ & & 16 & Retrospective & Multicen & ter & Manuscript & Cohort & 12 & & 65.5 \\
\hline Will et al. ${ }^{[18]}$ & & 15 & Prospective & Single & & Manuscript & Cohort & 9 & & 67 \\
\hline Dhir et al. ${ }^{[19]}$ & & 15 & Retrospective & Multicen & ter & Manuscript & Cohort & 10 & & 66.7 \\
\hline Artifon et al. ${ }^{[20]}$ & & 15 & Prospective & Single & & Manuscript & $\mathrm{RCT}$ & 4 & & 66 \\
\hline Gupta et al. ${ }^{[21]}$ & & 14 & Retrospective & Multicen & ter & Manuscript & Cohort & 23 & & 67.3 \\
\hline Weilert ${ }^{[22]}$ & & 14 & Prospective & Single & & Manuscript & Cohort & 2 & & 67.4 \\
\hline Song et al. ${ }^{[23]}$ & & 14 & Prospective & Single & & Manuscript & Cohort & 2 & & 67 \\
\hline Poincloux et al. ${ }^{[24]}$ & & 15 & Retrospective & Single & & Manuscript & Cohort & 10 & & 70.8 \\
\hline Dhir et al. ${ }^{[25]}$ & & 14 & Retrospective & Multicen & ter & Manuscript & Cohort & 68 & & NA \\
\hline Kawakubo et al. ${ }^{[26]}$ & & 14 & Retrospective & Multicen & ter & Manuscript & Cohort & 6 & & 72 \\
\hline Park et al. ${ }^{[27]}$ & & 13 & Prospective & Single & & Manuscript & Cohort & 4 & & 64.9 \\
\hline Bapaye et al. ${ }^{[28]}$ & & 13 & Retrospective & Single & & Manuscript & Cohort & 2 & & 59.9 \\
\hline Dhir et al. ${ }^{[29]}$ & & 13 & Retrospective & Single & & Manuscript & Cohort & 3 & & 53.4 \\
\hline Khashab et al. ${ }^{[30]}$ & & 13 & Retrospective & Single & & Manuscript & Cohort & 3 & & 66.1 \\
\hline $\begin{array}{l}\text { Prachayakul and } \\
\text { Aswakul[31] }\end{array}$ & & 13 & Retrospective & Single & & Manuscript & Cohort & 2 & & 62.8 \\
\hline Vila et al. ${ }^{[32]}$ & & 12 & Retrospective & Multicen & ter & Manuscript & Cohort & 12 & & 69.03 \\
\hline Kim et al. ${ }^{[33]}$ & & 12 & Retrospective & Multicen & ter & Manuscript & Cohort & 1 & & 68.8 \\
\hline Shah et al..$^{[34]}$ & & 11 & Retrospective & Single & & Manuscript & Cohort & $8 \varepsilon$ & & 65 \\
\hline & Males & Females & $\begin{array}{c}\text { Total } \\
\text { number of } \\
\text { procedures }\end{array}$ & $\begin{array}{l}\text { Technical } \\
\text { success }\end{array}$ & $\begin{array}{l}\text { Clinical } \\
\text { success }\end{array}$ & $\begin{array}{cc}\text { Total } & \text { Pne } \\
\text { adverse } & \\
\text { effects } & \end{array}$ & noperitoneum & Infection & Bleeding & $\begin{array}{l}\text { Bile } \\
\text { leak }\end{array}$ \\
\hline Minaga et al..$^{[12]}$ & 24 & 23 & 54 & 46 & 46 & 10 & 0 & 3 & 0 & 1 \\
\hline Park et al..$^{[13]}$ & 9 & 5 & 14 & 13 & 13 & 2 & 0 & 0 & 0 & 0 \\
\hline Tsuchiya et al. ${ }^{[14]}$ & 12 & 7 & 19 & 19 & 18 & 7 & 1 & 2 & 0 & 0 \\
\hline Kahaleh et al. ${ }^{[15]}$ & 16 & 19 & 35 & 32 & 31 & 9 & 0 & 2 & 2 & 0 \\
\hline Tyberg et al..$^{[16]}$ & 27 & 25 & 52 & 50 & 40 & 5 & 0 & 1 & 4 & 0 \\
\hline Khashab et al. ${ }^{[17]}$ & 70 & 51 & 121 & 112 & 93 & 20 & 1 & 7 & 2 & 3 \\
\hline Will et al. ${ }^{[18]}$ & 45 & 49 & 94 & 80 & 79 & 15 & 0 & 2 & 1 & 0 \\
\hline Dhir et al. ${ }^{[19]}$ & 58 & 46 & 104 & 97 & 93 & 9 & 0 & 1 & 2 & 2 \\
\hline Artifon et al. ${ }^{[20]}$ & 25 & 24 & 49 & 46 & 39 & 8 & 0 & 1 & 4 & 1 \\
\hline Gupta et al. ${ }^{[21]}$ & 119 & 115 & 234 & 207 & NA & 81 & 12 & 8 & 26 & 27 \\
\hline Weilert ${ }^{[22]}$ & 12 & 9 & 21 & 20 & 19 & 2 & 0 & 0 & 0 & 1 \\
\hline Song et al. ${ }^{[23]}$ & 13 & 14 & 27 & 27 & 26 & 5 & 3 & 0 & 1 & 0 \\
\hline Poincloux et al. ${ }^{[24]}$ & 58 & 43 & 101 & 99 & 93 & 12 & 2 & 3 & 1 & 5 \\
\hline Dhir et al. ${ }^{[25]}$ & 34 & 34 & 68 & 65 & 65 & 14 & 0 & 5 & 1 & 4 \\
\hline Kawakubo et al. ${ }^{[26]}$ & 35 & 29 & 64 & 61 & NA & 12 & 1 & 1 & 2 & 5 \\
\hline Park et al..$^{[27]}$ & 28 & 17 & 45 & 41 & 39 & 4 & 1 & 1 & 0 & 0 \\
\hline Bapaye et al. ${ }^{[28]}$ & 13 & 12 & 25 & 23 & 23 & 5 & 0 & 1 & 0 & 4 \\
\hline Dhir et al. ${ }^{[29]}$ & 22 & 13 & 35 & 34 & 34 & 12 & 2 & 0 & 0 & 2 \\
\hline Khashab et al. ${ }^{[30]}$ & 18 & 17 & 35 & 33 & 32 & 4 & 1 & 1 & 0 & 0 \\
\hline Prachayakul et al. ${ }^{[31]}$ & 11 & 10 & 21 & 20 & 19 & 2 & 1 & 0 & 0 & 1 \\
\hline Vila et al. ${ }^{[32]}$ & 71 & 54 & 125 & 84 & 79 & 29 & 0 & 1 & 6 & 0 \\
\hline Kim et al. ${ }^{[33]}$ & 9 & 4 & 13 & 12 & 11 & 5 & 0 & 2 & 0 & 0 \\
\hline Shah et al..$^{[34]}$ & 45 & 43 & 88 & 62 & 62 & 6 & 1 & 1 & 1 & 1 \\
\hline
\end{tabular}

RCT: Randomized controlled trial, NA: Not available 


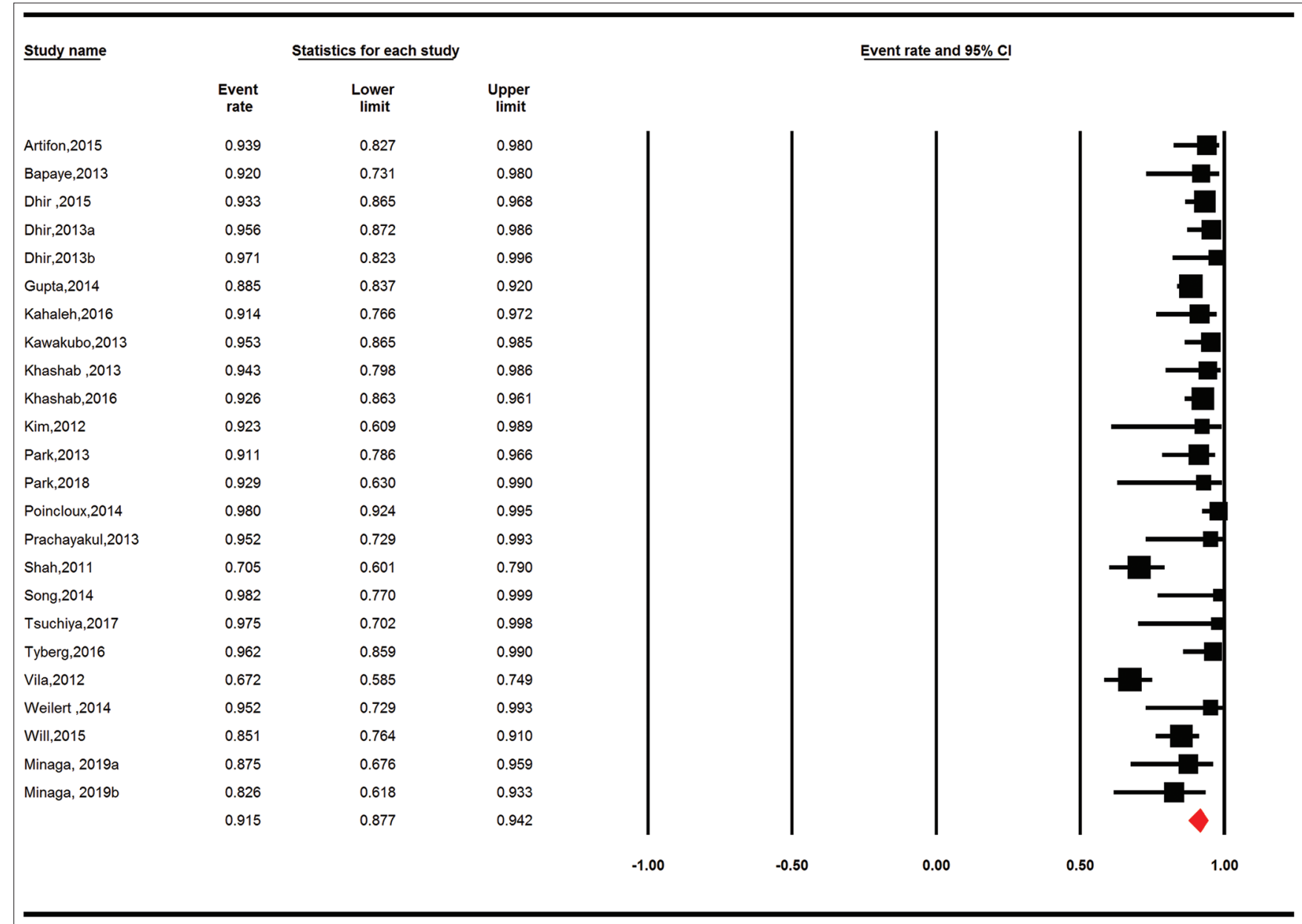

Figure 1. Forest plot - Technical success

\section{Re-intervention and adverse events}

The calculated pooled rate of re-intervention was 6.5\% (95\% CI: 3.8-10.8, $I^{2}=69.3$ ) [Figure 3].

The calculated pooled rate of adverse events was 17.9\% (95\% CI: 14.3-22.2, $\left.I^{2}=69.1\right)$ [Figure 4].

All results along with the adverse-event subtypes are summarized in Supplementary Figures 2-6.

At this stage, the authors decided to conduct a subgroup analysis on the data to explore reasons for the observed heterogeneity. Studies were subgrouped based on the study center (single center $v$ s. multicenter) and study type (prospective vs. retrospective; RCT $v s$. observational). The observed heterogeneity was not explained by this subgrouping. We were not able to subgroup and combine the studies based on the approach to BD (gastric vs. duodenal) due to the fact that the majority of the studies used a combination of these approaches.
Validation of meta-analysis results

Sensitivity analysis

To assess whether anyone study had a dominant effect on the meta-analysis, we excluded one study at a time and analyzed its effect on the main summary estimate. In this analysis, no single study significantly affected the outcome or the heterogeneity.

\section{Heterogeneity}

Based on Q statistics and $I^{2}$ analysis for heterogeneity, substantial heterogeneity $\left(I^{2}=69.1\right)$ was noted in the analysis of all adverse events, none $\left(I^{2}=0\right)$ with infection, moderate $\left(I^{2}=46.7\right)$ with bile leak, moderate $\left(I^{2}=41.8\right)$ with bleeding, low with pneumo-peritoneum $\left(I^{2}=2\right)$, and moderate with stent migration $\left(I^{2}=43.5\right)$.

Substantial heterogeneity $\left(I^{2}=76.5\right)$ was noted with the analysis of technical success and clinical success $\left(I^{2}=72.4\right)$. 


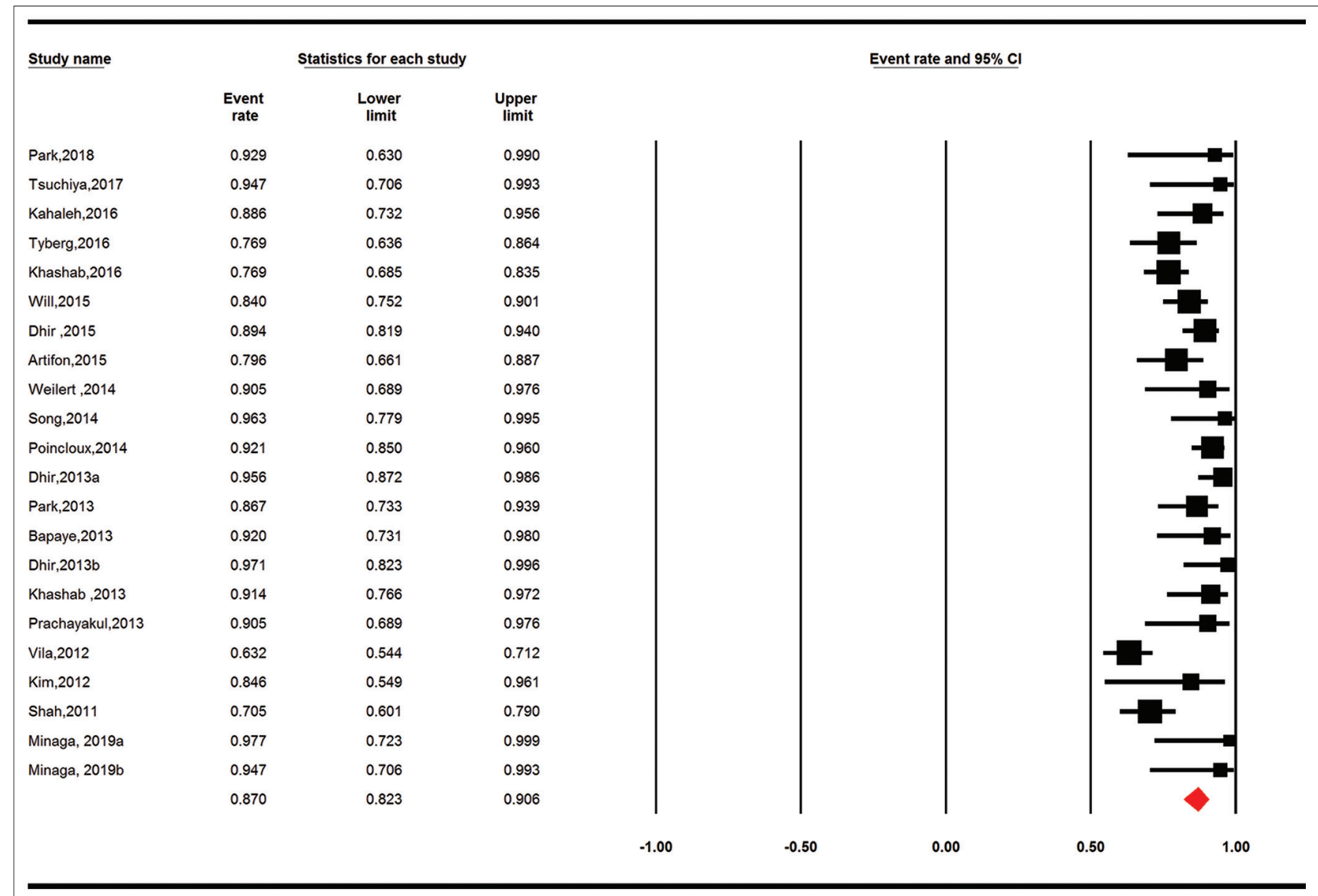

Figure 2. Forest plot - Clinical success

\section{Publication bias}

Based on the visual inspection of the funnel plot [Supplementary Figure 7] as well as the quantitative measurement that used the Egger regression test, there was evidence of publication bias. Further statistics using the fail-Safe $\mathrm{N}$ test and Duval and Tweedie's "Trim and Fill" test revealed the impact of the possible publication bias to be minimal and not to change the calculated estimate or the conclusion of this meta-analysis.

\section{DISCUSSION}

Based on the meta-analysis of EUS-guided gallbladder drainage done in 1437 patients from 23 studies, we report a pooled technical success rate of $91.5 \%$, a pooled clinical success rate of $87 \%$, and a pooled adverse event rate of $17.9 \%$.

ERCP remains the preferred procedure for providing internal BD. EUS-BD is utilized in patients who need biliary decompression when ERCP has either failed or is technically not feasible due to an inaccessible papilla and/or surgically altered anatomy and/or malignancy. The reported success and safety of EUS-BD have been variable across many studies. We report a pooled technical success rate of $91.5 \%$ with EUS-BD, and our calculated rate is on par with the currently reported values in literature, although with a heterogeneity percentage of $76.5 \%$, which may be due to one or more of the following reasons: (1) different techniques to access routes the gallbladder, including hepatogastrostomy (EUS-HG), cholecystostomy, choledochoduodenostomy (EUS-CDD), and other techniques; (2) use of different modalities of drainage, such as plastic stents, metal stents, lumen-apposing metal stents, nasobiliary drainage tubes, and a combination of these; and (3) the steep learning curve with the use of EUS in BD with accumulating experience. In the study by Vila et al., ${ }^{[32]}$ endoscopists with $>500$ EUS procedures had higher success rates than endoscopists who had performed fewer than 500 EUS procedures.

We report a clinical success rate of $87 \%$ with EUS-BD. Clinical success has also been variable across many studies, with reported clinical success in the range of 


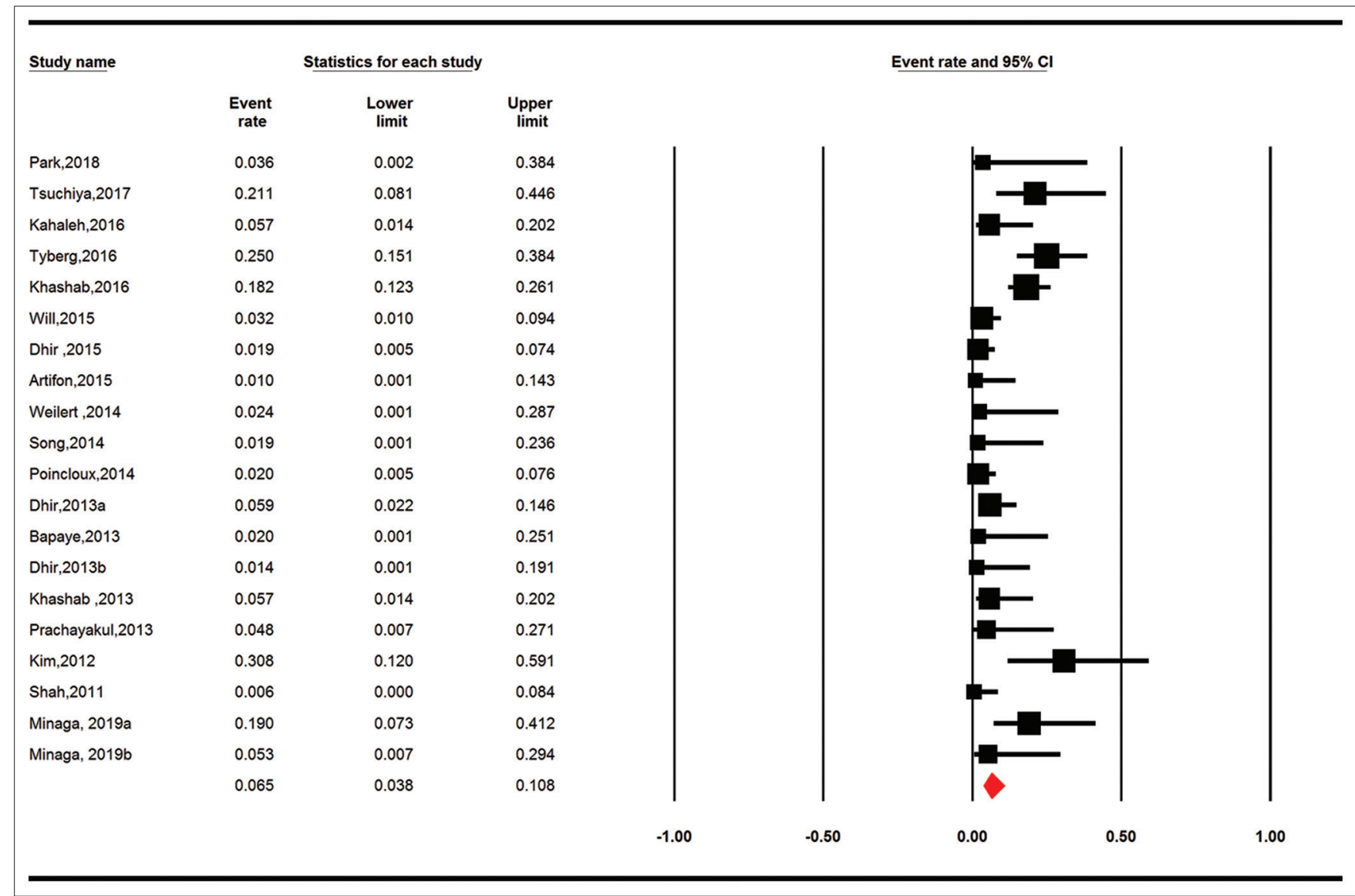

Figure 3. Forest plot - Reintervention

$63.2 \%-76.9 \%{ }^{[16,17,32,34]}$ Other studies have shown clinical success in the range of $84 \%-97 \% \cdot{ }^{[13,14,18,19,22-25,27-31,33]}$ In the majority of studies, ${ }^{[13-15,18,19,22-25,27-34]}$ clinical success has been closely related to technical success, indicating the importance of a successful procedure. A recent randomized controlled trial by Park et al. ${ }^{[13]}$ compared EUS- and ERCP-guided BD and showed no significant difference in technical and clinical success, with similar adverse events. However, it was a single-center study with a small population size.

Our analysis of the adverse events demonstrated an overall pooled rate of $17.9 \%$. The most commonly reported adverse events were biliary leak and infection. The pooled rate of biliary leaks was $4 \%$, and the pooled rate of infection and stent migration was $3.8 \%$. Our reported rates of the adverse events with EUS-BD are the key findings of this analysis, as studies vary widely in the reported rates of adverse events with EUS-BD. A recently published meta-analysis of studies ${ }^{[35]}$ reporting on EUS-guided CDD reported an overall pooled adverse event rate of $14.5 \%$ with EUS-CDD and $20.9 \%$ with EUS-HG. ${ }^{[35]}$ The overall adverse events were higher as compared to ERCP, which are reported to be $9.8 \%$ as per Enochsson et al. ${ }^{[1]}$

Our analysis of the subgroups, based on the study type (prospective vs. retrospective) and study center (single vs. multi), showed comparable technical success and clinical success rates in EUS-BD. We, however, noticed a reduction in the heterogeneity $I^{2}$ values with prospectively done studies as compared to the retrospective ones, suggesting that this could be one another contributing factor to our overall observed heterogeneity. We noted that the pooled rate of adverse events reported from multicenter studies was statistically higher when compared to single-center studies $(28 \%$ vs. $14 \%, P=0.02)$. The pooled re-intervention rate was numerically higher with multicenter-based studies as compared to single-center ones $(11 \%$ vs. $4 \%, P=0.07)$ and approached statistical significance.

The strengths of this review are as follows: systematic literature search with well-defined inclusion criteria, carefully excluding redundant studies, detailed extraction 


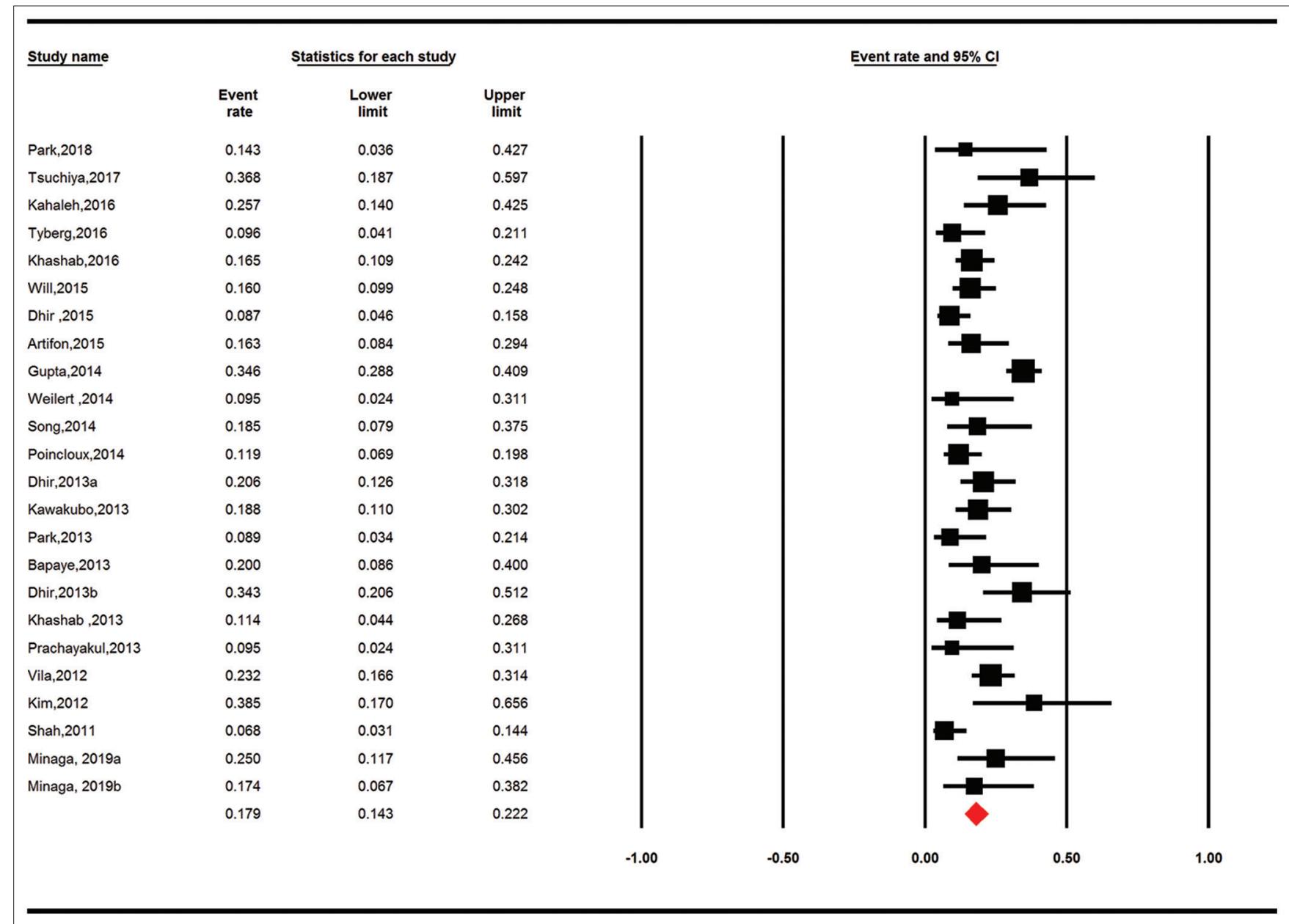

Figure 4. Forest plot - All adverse events

of adverse events, their subcategories, technical success, and clinical success information.

There are limitations to this study. The included studies were not entirely representative of the general population and community practice, with most studies being performed in tertiary-care referral centers. Our pooled rates were limited by heterogeneity. Although our subgroup analysis did not ascertain the cause for the observed heterogeneity, we believe that the most likely reason is the route of access to BD. Patient-related characteristics such as age, smoking, previous attempts and procedures, the stage of malignancy, and performance characteristics could influence the calculated rates. However, this estimate is the best available that summarizes the overall clinical outcomes with EUS-BD that is being rapidly adopted across centers around the world.

\section{CONCLUSION}

EUS-BD is a safe alternative option in cases where ERCP fails and in future may become an alternative to ERCP for BD. However, more well-conducted RCTs are needed to establish its role as an alternative to ERCP.

\section{Supplementary materials}

Supplementary information is linked to the online version of the article on the Endoscopic Ultrasound website.

Financial support and sponsorship

Nil.

\section{Conflicts of interest}

There are no conflicts of interest.

\section{REFERENCES}

1. Enochsson L, Swahn F, Arnelo U, et al. Nationwide, population-based data from 11,074 ERCP procedures from the Swedish registry for gallstone surgery and ERCP. Gastrointest Endosc 2010;72:1175-84, 1184.e1-3.

2. Giovannini M, Moutardier V, Pesenti C, et al. Endoscopic ultrasound-guided bilioduodenal anastomosis: A new technique for biliary drainage. Endoscopy 2001;33:898-900.

3. Stang A. Critical evaluation of the Newcastle-Ottawa scale for the assessment of the quality of nonrandomized studies in meta-analyses. Eur J Epidemiol 2010;25:603-5. 
4. DerSimonian R, Laird N. Meta-analysis in clinical trials. Control Clin Trials 1986;7:177-88.

5. Sutton AJ, Abrams KR, Jones DR, et al. Methods for Meta-Analysis in Medical Research. New York: John Wiley and Sons Ltd.; 2000. p. 205-8.

6. Higgins JP, Thompson SG, Deeks JJ, et al. Measuring inconsistency in meta-analyses. BMJ 2003;327:557-60.

7. Kanwal F, White D. "Systematic reviews and meta-analyses" in clinical gastroenterology and hepatology. Clin Gastroenterol Hepatol 2012;10:1184-6.

8. Guyatt GH, Oxman AD, Kunz R, et al. GRADE guidelines: 7. rating the quality of evidence-inconsistency. J Clin Epidemiol 2011;64:1294-302.

9. Easterbrook PJ, Berlin JA, Gopalan R, et al. Publication bias in clinical research. Lancet 1991;337:867-72.

10. Duval S, Tweedie R. Trim and fill: A simple funnel-plot-based method of testing and adjusting for publication bias in meta-analysis. Biometrics 2000;56:455-63.

11. Rothstein HR, Sutton AJ, Borenstein M. Publication bias in Meta-Analysis: Prevention, Assessment and Adjustments. West Sussex, England: John Wiley \& Sons,; 2006.

12. Minaga $\mathrm{K}$, Ogura $\mathrm{T}$, Shiomi $\mathrm{H}$, et al. Comparison of the efficacy and safety of endoscopic ultrasound-guided choledochoduodenostomy and hepaticogastrostomy for malignant distal biliary obstruction: Multicenter, randomized, clinical trial. Dig Endosc 2019;31:575-82.

13. Park JK, Woo YS, Noh DH, et al. Efficacy of EUS-guided and ERCP-guided biliary drainage for malignant biliary obstruction: Prospective randomized controlled study. Gastrointest Endosc 2018;88:277-82.

14. Tsuchiya $\mathrm{T}$, Teoh AY, Itoi $\mathrm{T}$, et al. Long-term outcomes of EUS-guided choledochoduodenostomy using a lumen-apposing metal stent for malignant distal biliary obstruction: A prospective multicenter study. Gastrointest Endosc 2018;87:1138-46.

15. Kahaleh M, Perez-Miranda M, Artifon EL, et al. International collaborative study on EUS-guided gallbladder drainage: Are we ready for prime time? Dig Liver Dis 2016;48:1054-7.

16. Tyberg A, Desai AP, Kumta NA, et al. EUS-guided biliary drainage after failed ERCP: A novel algorithm individualized based on patient anatomy. Gastrointest Endosc 2016;84:941-6.

17. Khashab MA, Messallam AA, Penas I, et al. International multicenter comparative trial of transluminal EUS-guided biliary drainage via hepatogastrostomy vs. choledochoduodenostomy approaches. Endosc Int Open 2016;4:E175-81.

18. Will U, Fueldner F, Kern C, et al. EUS-guided bile duct drainage (EUBD) in 95 patients. Ultraschall Med 2015;36:276-83.

19. Dhir V, Itoi $\mathrm{T}$, Khashab MA, et al. Multicenter comparative evaluation of endoscopic placement of expandable metal stents for malignant distal common bile duct obstruction by ERCP or EUS-guided approach. Gastrointest Endosc 2015;81:913-23.

20. Artifon EL, Marson FP, Gaidhane M, et al. Hepaticogastrostomy or choledochoduodenostomy for distal malignant biliary obstruction after failed ERCP: Is there any difference? Gastrointest Endosc 2015;81:950-9.
21. Gupta K, Perez-Miranda M, Kahaleh M, et al. Endoscopic ultrasound-assisted bile duct access and drainage: Multicenter, long-term analysis of approach, outcomes, and complications of a technique in evolution. J Clin Gastroenterol 2014;48:80-7.

22. Weilert F. Prospective evaluation of simplified algorithm for EUS-guided intra-hepatic biliary access and anterograde interventions for failed ERCP. Surg Endosc 2014;28:3193-9.

23. Song TJ, Lee SS, Park DH, et al. Preliminary report on a new hybrid metal stent for EUS-guided biliary drainage (with videos). Gastrointest Endosc 2014;80:707-11.

24. Poincloux L, Rouquette O, Buc E, et al. Endoscopic ultrasound-guided biliary drainage after failed ERCP: Cumulative experience of 101 procedures at a single center. Endoscopy 2015;47:794-801.

25. Dhir V, Artifon EL, Gupta K, et al. Multicenter study on endoscopic ultrasound-guided expandable biliary metal stent placement: Choice of access route, direction of stent insertion, and drainage route. Dig Endosc 2014;26:430-5.

26. Kawakubo $\mathrm{K}$, Isayama $\mathrm{H}$, Kato $\mathrm{H}$, et al. Multicenter retrospective study of endoscopic ultrasound-guided biliary drainage for malignant biliary obstruction in Japan. J Hepatobiliary Pancreat Sci 2014;21:328-34.

27. Park DH, Jeong SU, Lee BU, et al. Prospective evaluation of a treatment algorithm with enhanced guidewire manipulation protocol for EUS-guided biliary drainage after failed ERCP (with video). Gastrointest Endosc 2013;78:91-101.

28. Bapaye A, Dubale N, Aher A. Comparison of endosonography-guided vs. percutaneous biliary stenting when papilla is inaccessible for ERCP. United European Gastroenterol J 2013;1:285-93.

29. Dhir V, Bhandari S, Bapat M, et al. Comparison of transhepatic and extrahepatic routes for EUS-guided rendezvous procedure for distal CBD obstruction. United European Gastroenterol J 2013;1:103-8.

30. Khashab MA, Valeshabad AK, Modayil R, et al. EUS-guided biliary drainage by using a standardized approach for malignant biliary obstruction: Rendezvous versus direct transluminal techniques (with videos). Gastrointest Endosc 2013;78:734-41.

31. Prachayakul V, Aswakul P. A novel technique for endoscopic ultrasound-guided biliary drainage. World J Gastroenterol 2013;19:4758-63.

32. Vila JJ, Pérez-Miranda M, Vazquez-Sequeiros E, et al. Initial experience with EUS-guided cholangiopancreatography for biliary and pancreatic duct drainage: A Spanish national survey. Gastrointest Endosc 2012;76:1133-41

33. Kim TH, Kim SH, Oh HJ, et al. Endoscopic ultrasound-guided biliary drainage with placement of a fully covered metal stent for malignant biliary obstruction. World J Gastroenterol 2012;18:2526-32.

34. Shah JN, Marson F, Weilert F, et al. Single-operator, single-session EUS-guided anterograde cholangiopancreatography in failed ERCP or inaccessible papilla. Gastrointest Endosc 2012;75:56-64.

35. Mohan BP, Shakhatreh M, Garg R, et al. Efficacy and Safety of endoscopic ultrasound-guided choledochoduodenostomy: A systematic review and meta-analysis. J Clin Gastroenterol 2019;53:243-50. 
Supplementary Table 1 . Study quality assessment

\begin{tabular}{|c|c|c|c|c|c|c|c|}
\hline \multirow[t]{2}{*}{ Author } & \multirow[t]{2}{*}{ Study type } & \multirow{2}{*}{$\begin{array}{l}\text { Cohort } \\
\text { case-control }\end{array}$} & \multirow[t]{2}{*}{ Year } & \multirow{2}{*}{$\begin{array}{l}\text { Number of } \\
\text { patients }\end{array}$} & \multicolumn{3}{|c|}{ Newcastle-Ottawa Scale } \\
\hline & & & & & Selection & Comparability & Outcome \\
\hline Tsuchiya & Prospective & Cohort & 2017 & 19 & *** & ** & *** \\
\hline Kahaleh & Retrospective & Cohort & 2016 & 35 & $* * *$ & $* *$ & $* * *$ \\
\hline Tyberg & Prospective & Cohort & 2016 & 52 & $* *$ & * & *** \\
\hline Khashab & Retrospective & Cohort & 2016 & 121 & $* * *$ & $* *$ & $* * *$ \\
\hline Will & Prospective & Cohort & 2015 & 94 & $* * *$ & $* *$ & $* * *$ \\
\hline Dhir & Retrospective & Cohort & 2015 & 104 & $* * *$ & $* *$ & $* * *$ \\
\hline Gupta & Retrospective & Cohort & 2014 & 234 & $* * *$ & $* *$ & $* *$ \\
\hline Weilert & Prospective & Cohort & 2014 & 21 & $* * *$ & $* *$ & $* *$ \\
\hline Song & Prospective & Cohort & 2014 & 27 & $* *$ & $* *$ & $* *$ \\
\hline Poincloux & Retrospective & Cohort & 2014 & 101 & $* * *$ & $* *$ & $* * *$ \\
\hline Dhir & Retrospective & Cohort & 2013 & 68 & $* * *$ & $* *$ & $* *$ \\
\hline Kawakubo & Retrospective & Cohort & 2013 & 64 & $* * *$ & $* *$ & $* * *$ \\
\hline Park & Prospective & Cohort & 2013 & 45 & $* * *$ & $* *$ & $* * *$ \\
\hline Bapaye & Retrospective & Cohort & 2013 & 25 & $* * *$ & * & $* *$ \\
\hline Dhir & Retrospective & Cohort & 2013 & 35 & $* * *$ & $* *$ & $* *$ \\
\hline Khashab & Retrospective & Cohort & 2013 & 35 & $* * *$ & $* *$ & $* * *$ \\
\hline Prachayakul & Retrospective & Cohort & 2013 & 21 & $* * *$ & $* *$ & $* * *$ \\
\hline Vila & Retrospective & Cohort & 2012 & 125 & $* * *$ & * & $* *$ \\
\hline Kim & Retrospective & Cohort & 2012 & 13 & $* * *$ & * & $* *$ \\
\hline Shah & Retrospective & Cohort & 2011 & 66 & $* *$ & * & $* *$ \\
\hline \multicolumn{8}{|c|}{ Jadad-Oxford score for RCT } \\
\hline Minaga & Prospective & RCT & 2019 & 47 & 2 & 1 & 1 \\
\hline Park & Prospective & $\mathrm{RCT}$ & 2018 & 14 & 2 & 0 & 1 \\
\hline Artifon & Prospective & $\mathrm{RCT}$ & 2015 & 49 & 2 & 0 & 1 \\
\hline
\end{tabular}

*meaning: number of stars awarded to each criteria based on the quality of bias. RCT: Randomized controlled trial

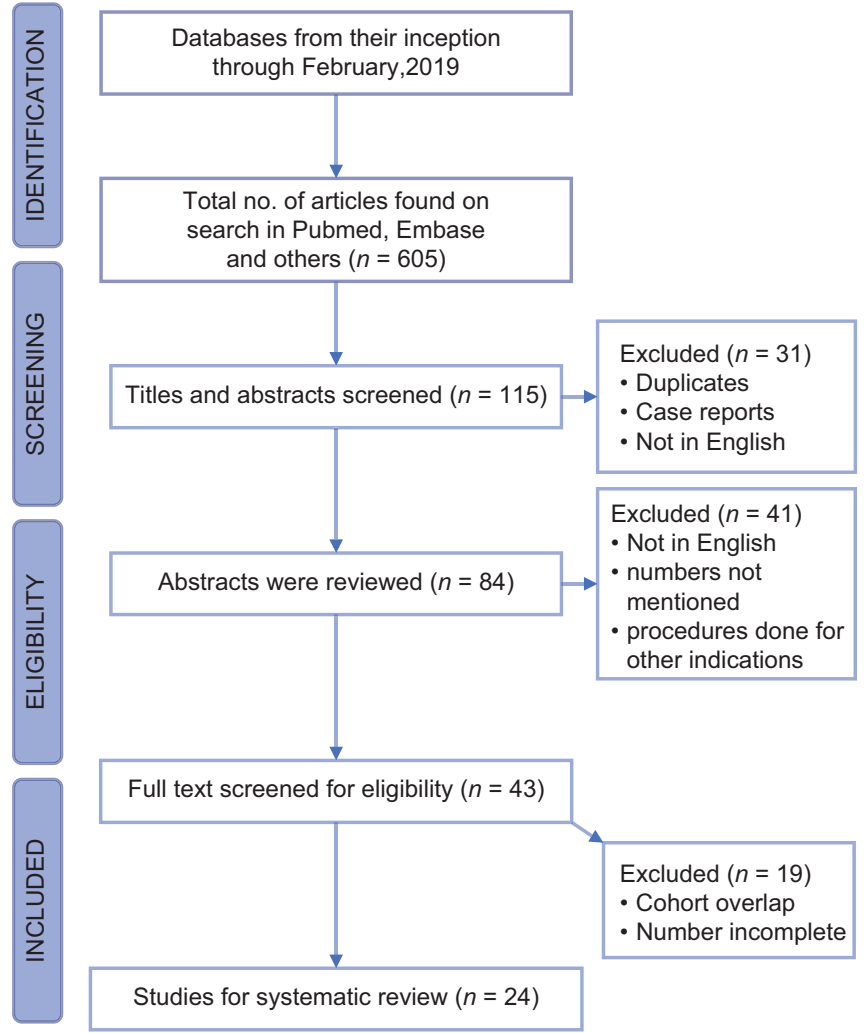

Supplementary Figure 1. Preferred Reporting items for Systematic Reviews and Meta-Analyses flow diagram of the study selection 


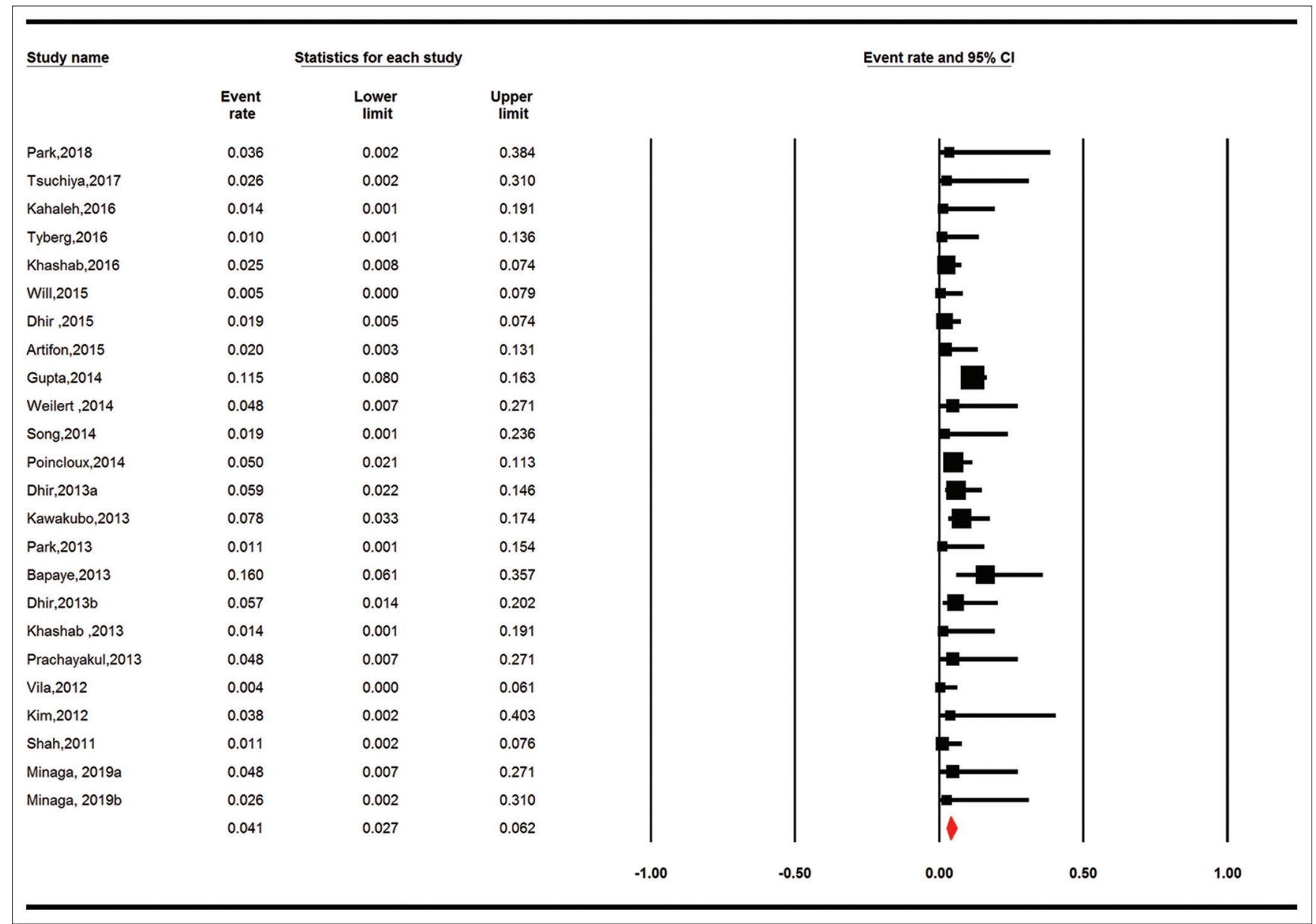

Supplementary Figure 2. Bile leak 


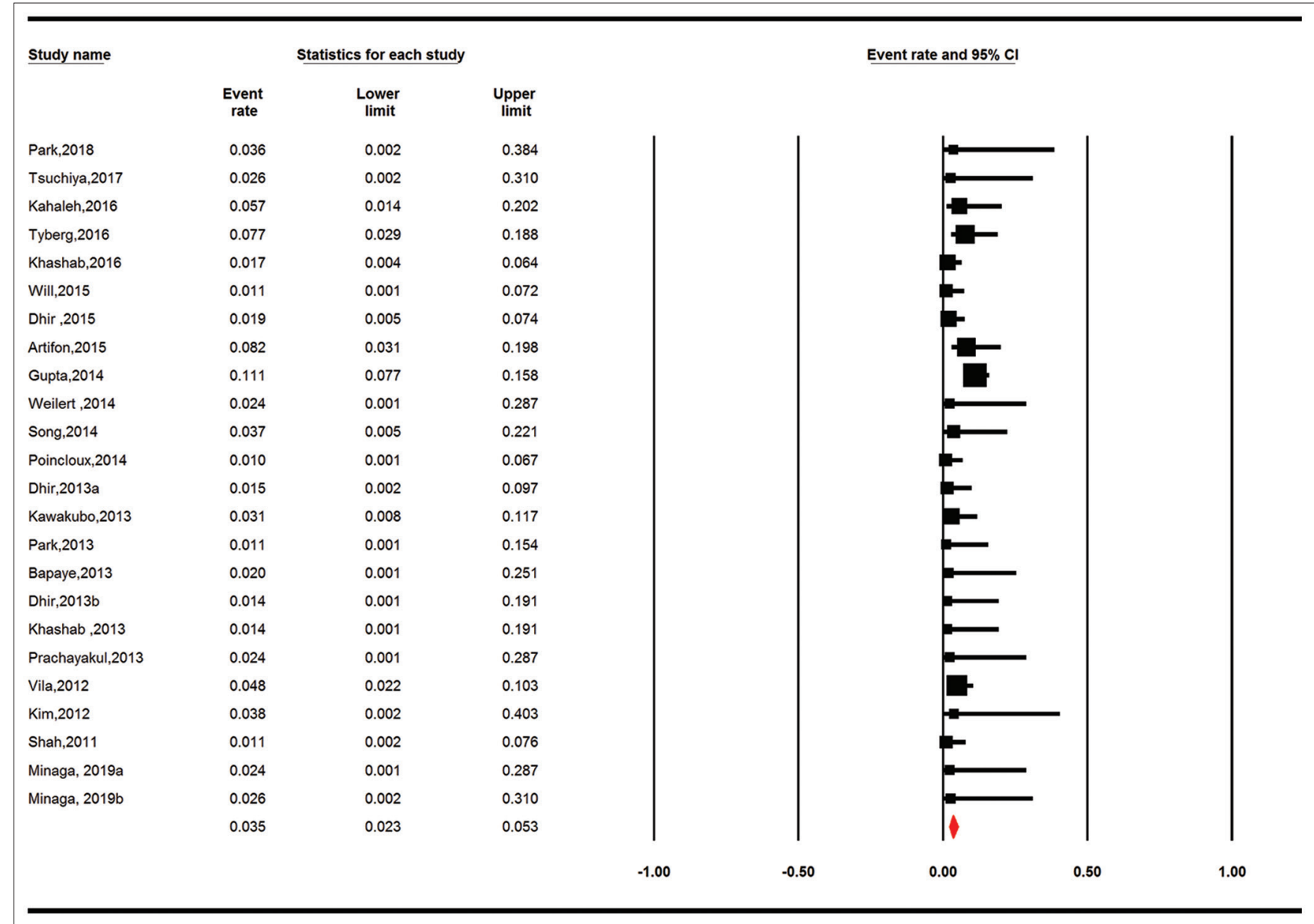

Supplementary Figure 3. Bleeding 


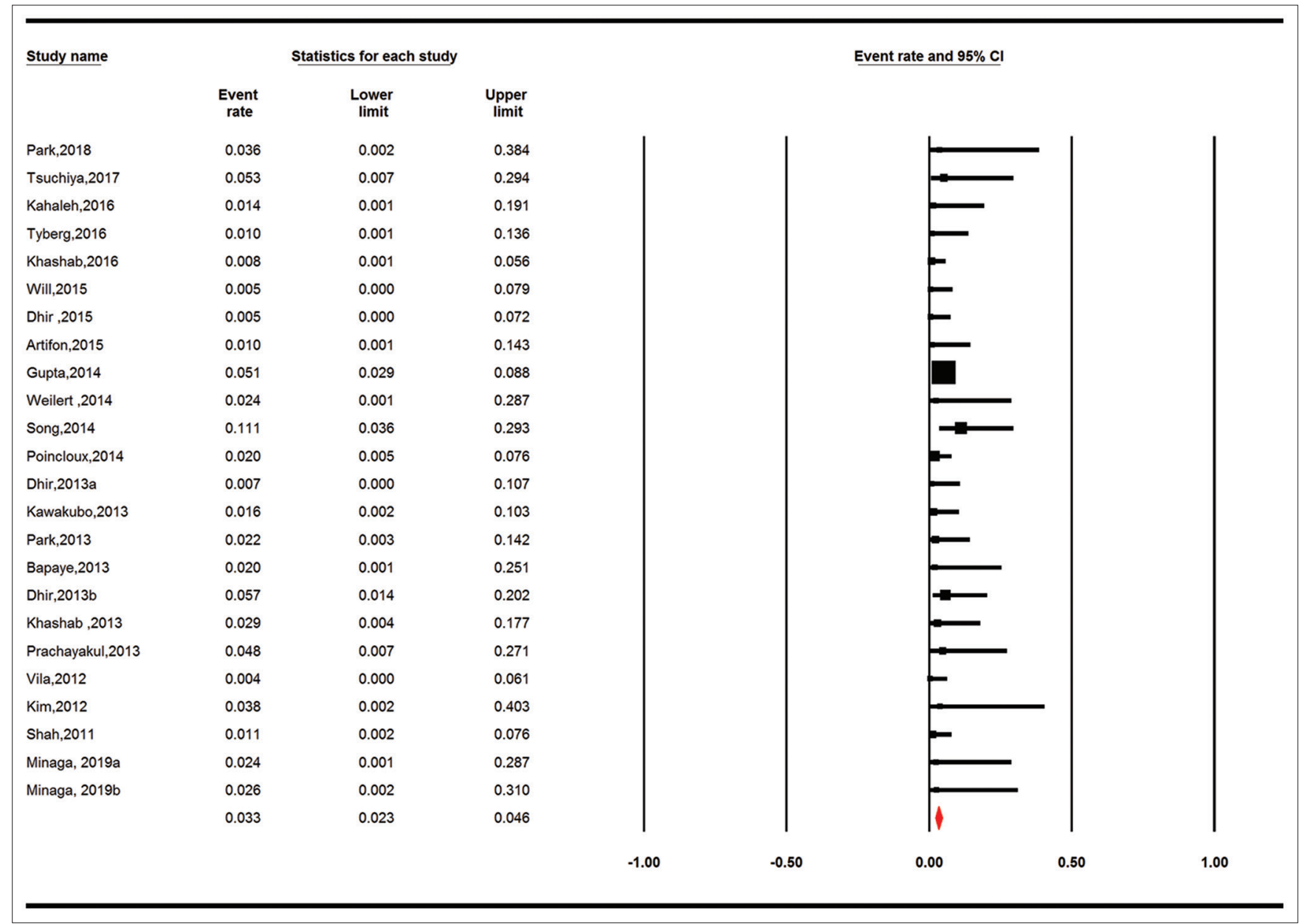

Supplementary Figure 4. Pneumoperitoneum 


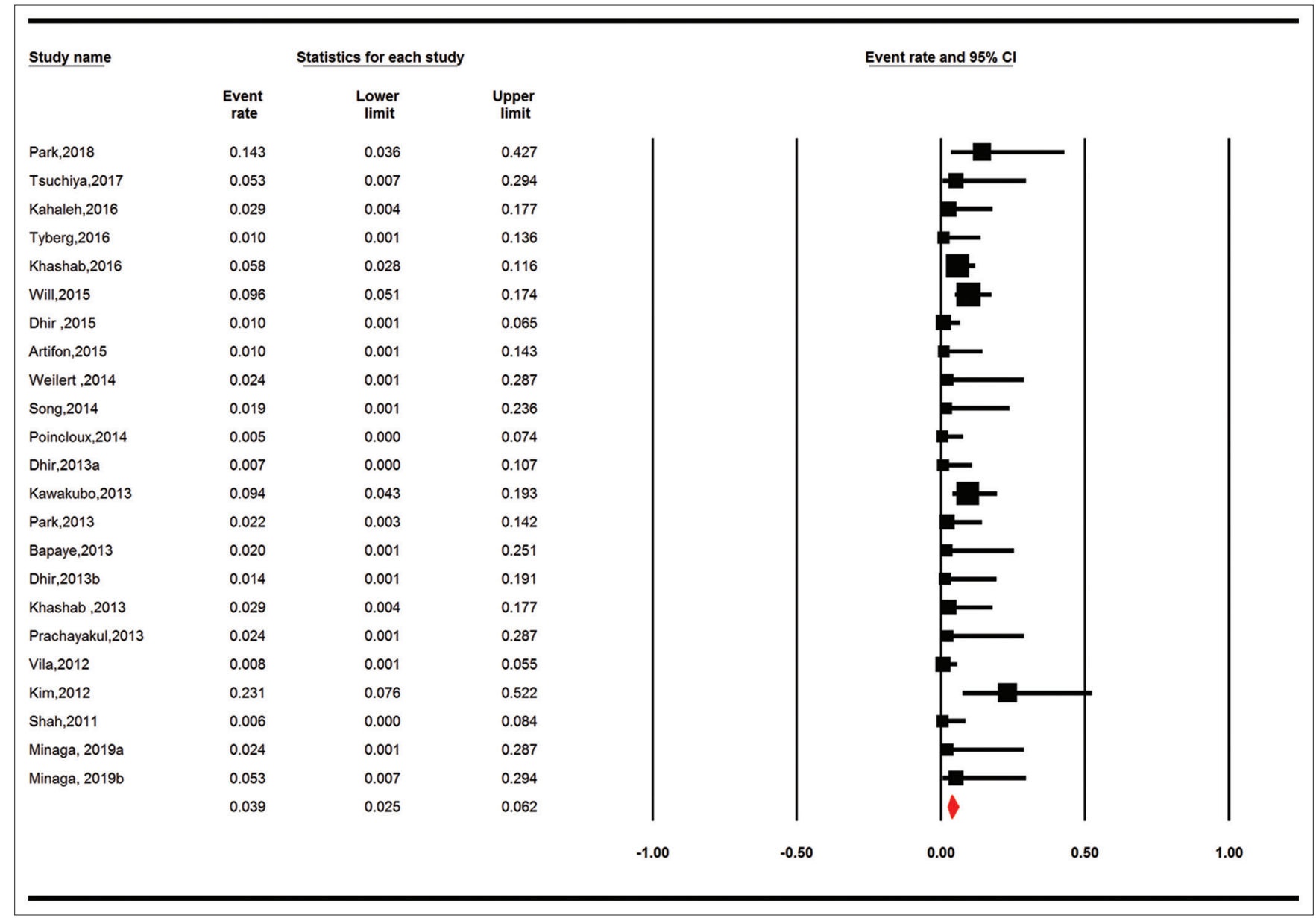

Supplementary Figure 5. Stent migration 


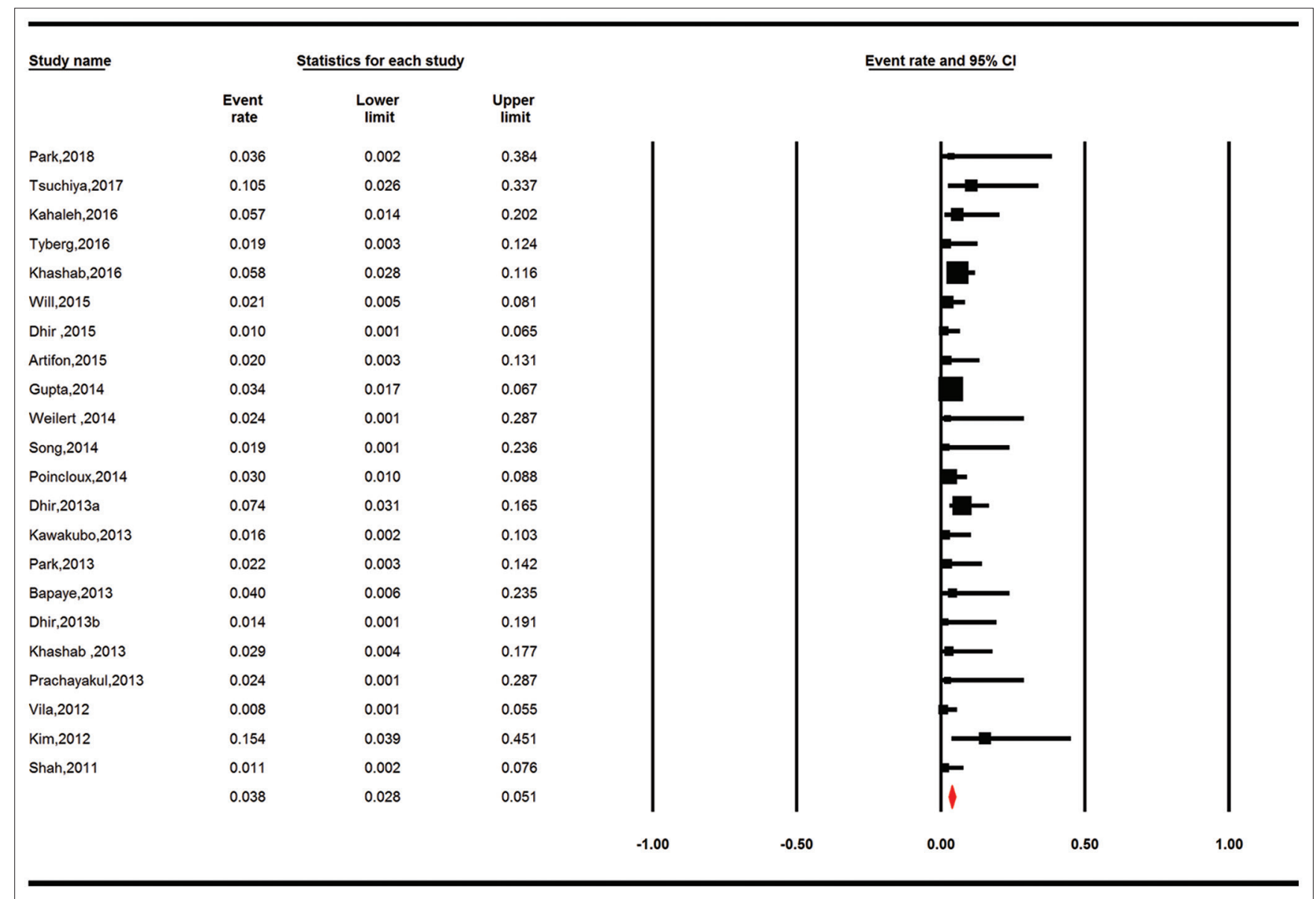

Supplementary Figure 6. Infection

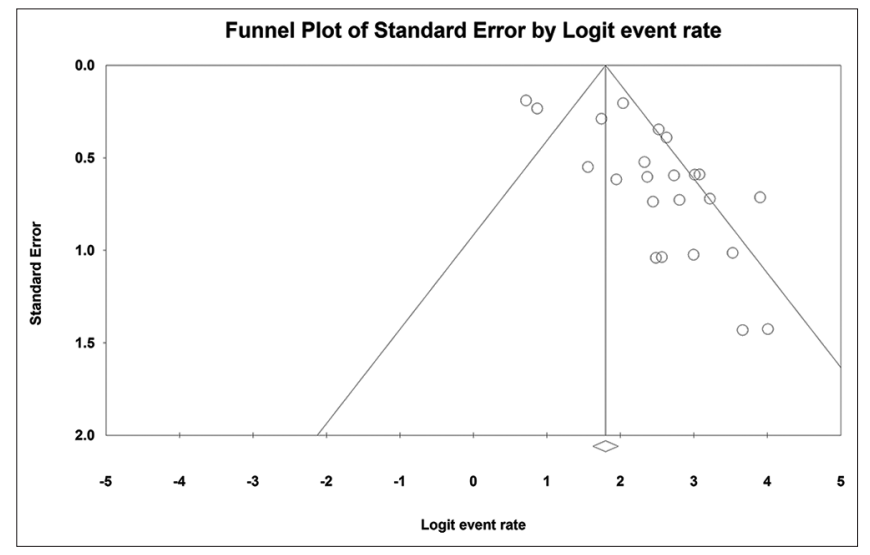

Supplementary Figure 7. Funnel plot 